\section{Nicht im Sinne des Erfinders}

1986 hat R. J. Simes im Journal of Clinical Oncology auf das Problem hingewiesen, dass Berichte über Forschung mit positiven oder vielversprechenden Resultaten eher publiziert werden als negative Resultate, was zu einem verzerrenden «publication bias» führen könne. Das «International Committee of Medical Journal Editors» (ICMJE) hat 2004 vorgeschlagen, dass die Registrierung in einem öffentlich zugänglichen Studienregister vor Studienbeginn als Bedingung für die Publikation einer Studie in den ICMJE-Mitgliederzeitschriften gelten solle. 2008 wurde diese Anforderung in die 7. Revision der Helsinki-Deklaration aufgenommen: «Jedes Forschungsvorhaben, an dem Versuchspersonen beteiligt sind, ist vor der Rekrutierung der ersten Versuchsperson in einer öffentlich zugänglichen Datenbank zu registrieren. ... Negative und nicht schlüssige Ergebnisse müssen ebenso wie positive veröffentlicht oder in anderer Form öffentlich verfügbar gemacht werden.» ${ }^{1}$

Eine kanadische Autorengruppe hat kürzlich in über 10'000 Artikeln aus 2105 Zeitschriften die Umsetzung dieser Forderung untersucht. ${ }^{2}$ In 71,2\% der Artikel war die «trial registration numben» mit dem Artikel publiziert worden, doch nur in $41,7 \%$ der Fälle war die Studie prospektiv, d.h. vor der Rekrutierung der Studienteilnehmenden, registriert worden.
Die Autorinnen und Autoren der Untersuchung vermuten, dass Studienverantwortliche eher dazu neigen, ihre Studie nachträglich zu registrieren, wenn eine Einreichung in einer Zeitschrift geplant wird. Diese Erkenntnisse bestätigen die ernüchternden Resultate früherer Untersuchungen: ${ }^{3,4}$ Selbst in angesehenen Zeitschriften mit hohem Impact Factor wurden häufig nicht registrierte Studien publiziert oder Studien, die erst nach einer möglichen Beobachtung der primären Endpunktdaten registriert worden waren.

In der ursprünglichen Diskussion um die Verpflichtung zur Registrierung klinischer Studien ging es unter anderem darum, selektive Berichterstattung und die dadurch entstehende Verzerrung der vorhandenen Evidenz zu verhindern. Die ICMJE weist auf ihrer Website sehr deutlich darauf hin, dass eine retrospektive Registrierung von Studien diesen Zweck nicht erfüllt.

Zehn Jahre nach der Revision der Helsinki-Deklaration scheint die Umsetzung dieser Ziele nur in sehr bescheidenem Ausmass gelungen zu sein.

Natalie Marty

1 WMA: Deklaration von Helsinki (2013). https://pkweb.ch/helsinki

2 Al-Durra M et al.. BMJ. 2020 Apr 14;369:m982.

3 Dal-Ré R et al. J Clin Epidemiol. 2016 Jul;75:100-7

4 Gopal AD et al. Trials. 2018 Aug 23;19:448

\author{
Nutzen Sie Ihr Abonnement optimal? \\ Ihr Abo umfasst nicht nur die 48 Seiten, die Sie als Print oder PDF erhalten! \\ Im Internet haben Sie ständig Zugriff auf \\ alle pharma-kritik-Jahrgänge seit 1988 \\ alle je veröffentlichten infomed-screen-Texte \\ alle je veröffentlichten Kurznachrichten («News»)
}

Wenn Sie den kostenlosen Mailing-Service abonnieren, bleiben Sie informiert

\title{
pharma-kritik
}

Herausgegeben von Etzel Gysling, gegründet 1979

in Zusammenarbeit mit Renato L. Galeazzi und Urs A. Meyer

«pharma-kritik» ist Mitglied der International Society of Drug Bulletins

Redaktionsteam: Renato L. Galeazzi, Etzel Gysling, Natalie Marty, Urspeter Masche, Peter Ritzmann, Alexandra Röllin, Stefan Weiler, Thomas Weissenbach

Layout und Sekretariat: Verena Gysling

Website: www.infomed.ch

E-Mail: sekretariat@infomed.ch

Infomed-Verlags-AG, Bergliweg 17, $9500 \mathrm{Wil}$

Telefon 071-910-0866, Telefax 071-910-0877

Abopreis für den Jahrgang 42 (2020): 92 Franken

Druck: Zehnder Print AG, 9500 Wil

(C) 2020 Infomed Wil. All rights reserved. ISSN 1010-5409 